\title{
Report of a Novel SHOX Missense Variant in a Boy With Short Stature and His Mother With Leri-Weill Dyschondrosteosis
}

\author{
Laura Lucchetti ${ }^{1}$, Paolo Prontera ${ }^{2}$, Amedea Mencarelli ${ }^{1,2}$, Ester Sallicandro ${ }^{2}$, \\ Annalisa Mencarelli', Marta Cofini ${ }^{1}$, Alberto Leonardi ${ }^{1}$, Gabriela Stangoni ${ }^{2}$, \\ Laura Penta ${ }^{1}$ and Susanna Esposito ${ }^{1 *}$ \\ ${ }^{1}$ Pediatric Clinic, Department of Surgical and Biomedical Sciences, Università degli Studi di Perugia, Perugia, \\ Italy, ${ }^{2}$ Medical Genetics Unit, Santa Maria della Misericordia Hospital, Perugia, Italy
}

OPEN ACCESS

Edited by:

Sandro Loche,

Ospedale Microcitemico,

Italy

Reviewed by:

Maurizio Delvecchio,

Ospedale Madonna Delle Grazie,

Italy

Stefano Zucchini,

Policlinico S. Orsola Malpighi,

Italy

${ }^{*}$ Correspondence:

Susanna Esposito

susanna.esposito@unimi.it

Specialty section:

This article was submitted to

Pediatric Endocrinology,

a section of the journal

Frontiers in Endocrinology

Received: 17 December 2017

Accepted: 27 March 2018

Published: 10 April 2018

Citation:

Lucchetti L, Prontera P, Mencarelli A, Sallicandro E, Mencarelli A, Cofini M, Leonardi A, Stangoni G, Penta L and Esposito S (2018) Report of a Novel SHOX Missense Variant in a Boy With Short Stature and

His Mother With Leri-Weill Dyschondrosteosis.

Front. Endocrinol. 9:163. doi: 10.3389/fendo.2018.00163
Heterozygous mutations in the SHOX gene or in the upstream and downstream enhancer elements are associated with 2-22\% of cases of idiopathic short stature (OMIM \#300582) and with $60 \%$ of cases of Leri-Weill dyschondrosteosis (OMIM \#127300) with which female subjects are generally more severely affected. Approximately $80-90 \%$ of SHOX pathogenic variants are deletions or duplications, and the remaining 10-20\% are point mutations that primarily give rise to missense variants. The clinical interpretation of novel variants, particularly missense variants, can be challenging and can remain of uncertain significance. Here, we describe a novel missense variant (c.1044 G>T, p.Arg118Met) in a Moroccan boy with a disproportionately short stature and without any radiological traits or bone deformities and in his mother, who had a disproportionately short stature and a Madelung deformity. This variant has not been reported to date in the updated SHOX allelic variant or Human Gene Mutation Databases nor is it listed as a polymorphism in the ExAC browser, dbSNP, or 1000G. This mutation was predicted to be deleterious by three different bioinformatics tools since it modifies an amino acid in a highly conserved DNA-binding domain of the SHOX protein. Based on this evidence, the patient was treated with recombinant human growth hormone.

Keywords: short stature, Leri-Weill dyschondrosteosis, novel missense mutation, SHOX, pediatric endocrinology

\section{BACKGROUND}

Human growth is a multifactorial phenomenon that is controlled by nutrition, environment, endocrine factors, and many genes. Although several genes seem to be associated with short stature, only a small proportion occurs sufficiently frequently to be recognized by clinicians and radiologists (1). These genes are responsible for bone disorders that cause incorrect balances of the proliferation and differentiation of chondrocytes and pathological extensions in length and width (1). The SHOX gene encodes a transcription factor with a common DNA-binding domain, a so-called homeodomain, which is implicated in skeletal development. Mutations in the SHOX gene are a possible cause of isolated or familial short stature $(2,3)$.

Homozygous or compound heterozygous mutations of the SHOX gene or its downstream enhancer cause $75 \%$ of Langer mesomelic dysplasia (OMIM \#249700), which is the more severe clinical form that includes a disproportionately short stature. Heterozygous mutations in SHOX or in the 
upstream and downstream enhancer elements are associated with $2-22 \%$ of cases of idiopathic short stature (ISS; OMIM \#300582) $(2,4-6)$ and with $60 \%$ of cases of Leri-Weill dyschondrosteosis (LWD; OMIM \#127300), which usually affects female subjects with severe manifestations (7). The most frequent mutation is the deletion of the entire or partial SHOX locus (i.e., $80-90 \%$ of cases), whereas point mutations appear to be less frequent $(10-20 \%)(5,8)$. The phenotype associated with heterozygous SHOX mutations is a continuum from milder short stature without radiological findings to disproportionately short stature with Madelung deformity. The clinical symptoms can be markedly different even among the affected members of the same family who have the same genetic defects $(1,3,9-12)$. This case describes a novel SHOX mutation in a Moroccan boy with disproportionate short stature and his mother with disproportionate short stature and LWD.

\section{CASE PRESENTATION}

\section{Clinical Report}

The Ethics Committee of Umbria Region (CEVAS) approved the study, and written informed consent was obtained from the parents of the enrolled child.

The patient is a 6-year-old Moroccan boy who was born full-term after an uncomplicated pregnancy with a birth weight
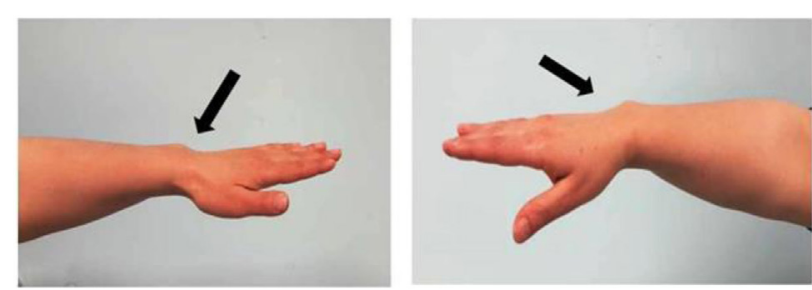

FIGURE 1 | Photograph of the forearm of the proband's mother showing a Madelung deformity and bowing of the radius (black and white arrows), which gives the hand and the wrist the appearance of a dinner fork. of $3,020 \mathrm{~kg}(-0.86 \mathrm{SDS})$ and a length of $50 \mathrm{~cm}(-0.1 \mathrm{SDS}) . \mathrm{He}$ was the only child of non-consanguineous parents. His mother's height $(\mathrm{H})$ was $148.9 \mathrm{~cm}(-2.82$ SDS), and his father's $\mathrm{H}$ was $172.5 \mathrm{~cm}(-0.39$ SDS) with a target $\mathrm{H}$ of $167.2(-1.13 \mathrm{SDS})$. The mother had a disharmonic short stature (sitting $\mathrm{H} / \mathrm{H}$ : 0.563 and SPAN/H: 0.953) and Madelung deformity of the forearm, which suggested a diagnosis of LWD (Figure 1).

When the patient was first referred to our Unit of Pediatric Endocrinology at the age of 2.9 years, he exhibited normal psychomotor development, his $\mathrm{H}$ was $86.5 \mathrm{~cm}$ (-1.90 SDS), his weight was $14 \mathrm{~kg}$ (50th percentile), and his body mass index (BMI) was 18.7 (1.38 SDS). The auxological data are expressed in SDS according to the population standard for age and gender. $\mathrm{He}$ was prepubertal with testes $<2 \mathrm{~mL}$ in volume. The pubertal stage was evaluated according to the Tanner-Marshall method. The physical examination was normal with no bone deformities or dysmorphism. The bone age of hand and wrist at a chronological age of 3 years conformed to the Greulich-Pyle atlas standard for 2.4 years, and an X-ray of the wrist and forearm ruled out severe radiological deformity except for a mild bowing of the radius (Figure 2).

We re-evaluated the child after 6 and 12 months. At 3.9 years, his height was $91.3(-2.20$ SDS) with a growth velocity of $5.30 \mathrm{~cm} /$ year (10th p; $-1.61 \mathrm{SDS}$ ). The Rappold's score was 6 with a pathological sitting $\mathrm{H} / \mathrm{H}$ ratio of 0.58 (normal value, $<0.55$ ) and a BMI > the 50th percentile. His arm SPAN/H ratio was normal ( 0.99 with a normal value $>0.965)$, and other parameters were negative. Laboratory investigations revealed a normal hemoglobin value, and normal kidney and hepatic functions and excluded celiac disease and thyroid dysfunction. His endocrine evaluation revealed a peak growth hormone value of $4.5 \mathrm{ng} /$ $\mathrm{mL}$ using arginine tests and $8.58 \mathrm{ng} / \mathrm{mL}$ using a clonidine test (normal value, $>8$ ) with a normal level of insulin-like growth factor-1 (IGF-1; $88 \mathrm{ng} / \mathrm{mL}, 50$ th-75th percentile), which ruled out a growth hormone deficit.

We examined the child again when he was 5.9 years old. His $\mathrm{H}$ was 1.99 SDS below the mean for age and sex $(104.9 \mathrm{~cm})$. The arm SPAN was $101.5 \mathrm{~cm}$ with a non-pathological arm SPAN/H ratio

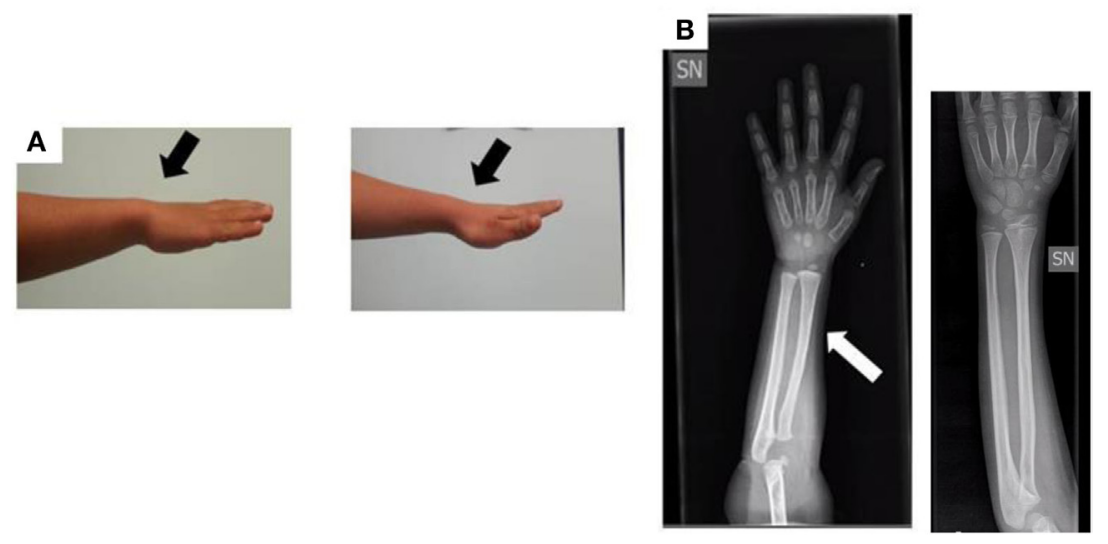

FIGURE 2 | (A) Photograph of the forearm of the proband. No Madelung deformity is present (black arrows). (B) Radiograph of the forearm of the proband at the age of 3 years: the white arrow shows a mild bowing of the radius in comparison to a normal radiograph (the one without arrow). 
of 0.97 . The sitting $\mathrm{H}$ was $59.9 \mathrm{~cm}$ with a distinctly supranormal sitting $\mathrm{H} / \mathrm{H}$ ratio of 0.57 .

\section{Molecular Studies}

Genomic DNA was extracted from the peripheral blood of the proband and his parents following standard procedures. Thereafter, multiplex ligation-dependent probe amplification (MLPA) was performed with an SALSA MLPA P018 SHOX-G1 probemix kit (MRC-Holland, Amsterdam, the Netherlands) in accordance with the manufacturer's recommendations. The results were analyzed with the Coffalyser software (MRC-Holland, Amsterdam, The Netherlands).

A polymerase chain reaction (PCR) was performed with specific oligonucleotides to amplify the SHOX-coding exons $(2,3,4,5$, and $6 \mathrm{a})$ and flanking intragenic regions. The PCR products were directly sequenced using a CEQ TM8000 Genetic Analysis System (Beckman Coulter, Brea, CA, USA). The results were analyzed by comparing the sequences with those reported in Gene Bank NG_009385.1 and NM_004551.3 and http://grenada.lumc.nl/LOVD2/MR/home.php?select_db=SHOX (Leiden Open Variation Database, Leiden, The Netherlands).

\section{Results}

No deletions were found through the MLPA analysis. The SHOX gene sequencing revealed the heterozygous missense variant c.1044 (c.1044 G>T) in exon 3 (Figure 3). This transversion was predicted to change the inside of the homeodomain sequence from an Arginine to a Methionine in codon 118 (p.Arg118Met). This variant was found in neither the ExAC nor $1000 \mathrm{G}$ databases. Moreover, it has not been described in the Human Gene Mutation Database, the Human Short Stature Gene Allelic Variant Database Web Site, or the X-chromosome gene database $(13,14)$. The same variant was found in his mother.

After the analysis of the molecular studies, therapy with recombinant human growth hormone (rh-GH) was started according to the Italian Drug Administration (AIFA) and an auxological follow-up was initiated. The starting rh-GH dose was $0.030 \mathrm{mg} / \mathrm{kg} /$ day.

To assess the effect of rh-GH therapy, we considered the difference in height SDS, height velocity both as SDS and as $\mathrm{cm} /$ years. After the first 12 months of treatment, a significant increase of height was evidenced, with a height gain of +0.40 SDS (Tanner growth charts). Also the height velocity got better reaching $7.23 \mathrm{~cm} /$ year corresponding to +2.16 SDS for age and sex, in comparison with -2.05 SDS before treatment.

\section{DISCUSSION}

This study describes the clinical and molecular data of a motherson pair who shared short stature and a novel missense SHOX variant. The novel point mutation described here has not been reported in the updated databases and has not been described by other authors in the literature. Consequently, the phenotypic effect of this mutation is not completely known. This variant segregates in the family reported here with short stature (proband) and LWD (mother), and the latter phenotype is very specific for SHOX pathogenic mutations. Moreover, this C-to-G transversion at nucleotide c.1044 (c.1044 G>T) in exon 3 is predicted to result in an amino acid alteration from Arginine to Methionine inside the homeodomain sequence in codon 118 (p.Arg118Met). This mutation affects a highly evolutionarily conserved amino acid of the homeodomain in the SHOX protein and seems to cause a loss of function by altering the secondary structure of the protein. Predictions from functional effect studies demonstrated that this mutation is probably damaging with a maximal score (PolyPhen2, SIFT, PROVEAN).

The homeodomain of SHOX gene mediates several key functions that include nuclear localization, DNA binding, and proteinprotein interactions (15); consequently, mutations located in this region may impair these processes and lead to bone defects $(1,5,8,16)$. For these reasons, most of the point mutations (i.e., missense or nonsense mutations) that lead to ISS and LWD

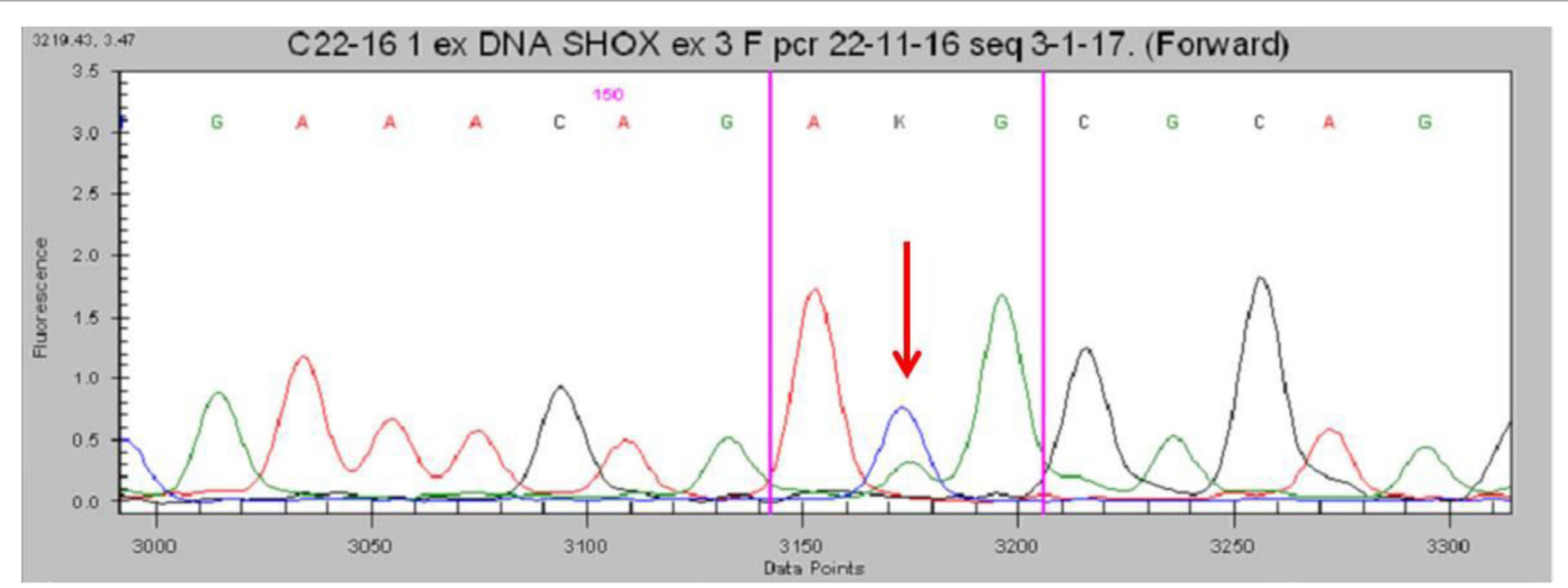

FIGURE 3 | Partial electropherogram of the Sanger sequencing of SHOX in the patient shows (red arrow) the c.1044G>T mutation. 
are located within exons 3 and 4, although they can be spread all over the gene $(1,8)$.

Furthermore, in the analysis of the Human Gene Mutation Database, we observed that 47 point mutations of all 88 mutations known to date are located in the areas of codons 116 and 176, which encode the homeodomain. Binder et al. described a novel missense mutation (p.Arg119Gly) located at the beginning of the homeobox (1). This mutation is a C-to-G transversion at nucleotide 355 in exon 3 that is predicted to result in a change from the charged polar amino acid Arginine to the non-polar amino acid Glycine. This mutation has been detected in two affected siblings and their mother. The siblings are a 15.9-year-old male with a disharmonic short stature ( -1.77 SDS) associated with a pathological sitting $\mathrm{H} / \mathrm{H}$ ratio (4.31 SDS) and a 13.3-year-old female with a severe short stature ( -2.40 SDS) and pathological sitting $\mathrm{H} / \mathrm{H}$ ratio (3.66 SDS). In 2002, Rappold et al. described a novel mutation located in the homeobox at codon 116. In this case, the mutation was responsible for LWD (2). In our patient, the p.ArgR118Met mutation was detected in codon 118, i.e., in the gene sequence that plays a key role in the correct function of the protein. In comparison with the previous cases, our patient has an disharmonic mild short stature ( -1.90 SDS) with a pathological sitting $\mathrm{H} / \mathrm{H}$ ratio (1 SDS), although his condition is probably less severe than usually reported because of his young age. Even in this case, as described in the literature for the other mutations, the main characteristics of mesomelic disproportions of the limbs and the Madelung deformity of the forearm will appear during the second decade of life $(1,3,17)$. Several studies have observed that the main characteristics of mesomelic disproportions of the limbs and the Madelung deformity of the forearm develop over time, appear during the second decade of life and, occasionally, the skeletal disproportions caused by SHOX mutations are not yet expressed in pre-school children, especially in boys (1). In addition, in our case, the mother, who had the same mutation, had a more severe short stature and the Madelung deformity. Hence, in our case we can assess that despite the proband and his mother have the same genetic mutation, the different phenotype can be probably due both to the different age and the rule of estrogen that in female affected patients seems to worsened the clinical findings.

The challenge of finding SHOX mutations is due to the possibility of the treatment of all patients with SHOX haploinsufficiencies with rh-GH therapy. Several studies have demonstrated that rh-GH therapy improves linear growth, results in a significant increase in height SDS that reaches the lower end of the normal range by the end of the second year of therapy and results in a

\section{REFERENCES}

1. Binder G. Short stature due to SHOX deficiency: genotype, phenotype, and therapy. Horm Res Paediatr (2011) 75:81-9. doi:10.1159/000324105

2. Rappold GA, Fukami M, Niesler B, Schiller S, Zumkeller W, Bettendorf M, et al. Deletions of the homeobox gene SHOX (short stature homeobox) are an important cause of growth failure in children with short stature. J Clin Endocrinol Metab (2002) 87:1402-6. doi:10.1210/jcem.87.3.8328

3. Rappold G, Blum WF, Shavrikova EP, Crowe BJ, Roeth R, Quigley CA, et al. Genotypes and phenotypes in children with short stature: clinical indicators of SHOX haploinsufficiency. J Med Genet (2007) 44:306-13. doi:10.1136/ jmg.2006.046581 significant increase in IGF-1 concentration (1). According to previously auxological data reported, also in our case a significant good response to the rh-GH therapy has been evidenced.

\section{CONCLUSION}

In conclusion, we found a novel point mutation that is associated with a disharmonic short stature without wrist or forearm deformities in the proband, inherited from his mother who showed LWD, a more convincing SHOX-related phenotype. In this case report, the genotype-phenotype correlation is not completely known, but the presence of the Madelung deformity associated with the same mutation in the proband's mother could suggest an association between this novel mutation and SHOX haploinsufficiency. This case underlines the importance for clinicians in the diagnostic approach to the short stature of evaluating also children's parents because some phenotypic characteristics of SHOX deficiency can develop over time.

\section{CONSENT TO PUBLISH STATEMENT}

The Ethics Committee of the University of Perugia approved the publication of this case, and both parents provided written informed consent for the publication of this manuscript.

\section{ETHICS STATEMENT}

This study was approved by the Ethics Committee of the University of Perugia, and both parents provided written informed consent for the evaluation of themselves and the child.

\section{AUTHOR CONTRIBUTIONS}

LL and PP wrote the first draft of the manuscript; AmM and ES performed the laboratory analyses; AnM, MC, and AL were in charge of the patient's follow-up; GS and LP performed the diagnosis; SE supervised the patient's management and critically revised the paper. All the authors read and approved the final version of the manuscript.

\section{FUNDING}

This study was partially support by a grant of the World Association for Infectious Diseases and Immunological Disorders (WAidid 2015_03).

4. Rao E, Weiss B, Fukami M, Rump A, Niesler B, Mertz A, et al. Pseudoautosomal deletions encompassing a novel homeobox gene cause growth failure in idiopathic short stature and Turner syndrome. Nat Genet (1997) 16:54-63. doi:10.1038/ng0597-54

5. Jorge AAL, Souza SC, Nishi MY, Billerbeck AE, Libório DCC, Kim CA, et al. SHOX mutations in idiopathic short stature and Leri-Weill dyschondrosteosis: frequency and phenotypic variability. Clin Endocrinol (Oxf) (2007) $66: 130-5$

6. Benito-Sanz S, Barroso E, Heine-Suñer D, Hisado-Oliva A, Romanelli V, Rosell J, et al. Clinical and molecular evaluation of SHOX/PAR1 duplications in Leri-Weill dyschondrosteosis (LWD) and idiopathic short stature (ISS). J Clin Endocrinol Metab (2011) 96:404-12. doi:10.1210/jc.2010-1689 
7. Ogata T, Matsuo N, Nishimura G. SHOX haploinsufficiency and overdosage: impact of gonadal function status. J Med Genet (2001) 38:1-6. doi:10.1136/ jmg.38.1.1

8. Benito-Sanz S, del Blanco DG, Aza-Carmona M, Magano LF, Lapunzina P, Argente J, et al. PAR1 deletions downstream of SHOX are the most frequent defect in a Spanish cohort of Léri-Weill dyschondrosteosis (LWD) probands. Hum Mutat (2006) 27:1062. doi:10.1002/humu.9456

9. Auger J, Baptiste A, Benabbad I, Thierry G, Costa JM, Amouyal M, et al. Genotype-phenotype relationship in patients and relatives with SHOX region anomalies in the French population. Horm Res Paediatr (2016) 86:309-18. doi:10.1159/000448282

10. Binder G, Schwarze CP, Ranke MB. Identification of short stature caused by SHOX defects and therapeutic effect of recombinant human growth hormone. J Clin Endocrinol Metab (2000) 85:245-9. doi:10.1210/jcem.85.1.6375

11. Binder G, Ranke MB, Martin DD. Auxology is a valuable instrument for the clinical diagnosis of SHOX haploinsufficiency in school-age children with unexplained short stature. J Clin Endocrinol Metab (2003) 88:4891-6. doi:10.1210/jc.2003-030136

12. Hirschfeldova K, Solc R, Baxova A, Zapletalova J, Kebrdlova V, Gaillyova R, et al. SHOX gene defects and selected dysmorphic signs in patients of idiopathic short stature and Leri-Weill dyschondrosteosis. Gene (2012) 491:123-7. doi:10.1016/j.gene.2011.10.011

13. Niesler B, Fischer C, Rappold GA. The human SHOX mutation database. Hum Mutat (2002) 30:386-94.

14. Niesler B, Röth R, Wilke S, Fujimura F, Fischer C, Rappold G. The novel human SHOX allelic variant database. Hum Mutat (2007) 28:933-8. doi:10.1002/ humu. 20542
15. Schneider KU, Marchini A, Sabherwal N, Röth R, Niesler B, Marttila T, et al. Alteration of DNA binding, dimerization, and nuclear translocation of SHOX homeodomain mutations identified in idiopathic short stature and Leri-Weill dyschondrosteosis. Hum Mutat (2005) 26:44-52. doi:10.1002/ humu. 20187

16. Chen J, Wildhardt G, Zhong Z, Röth R, Weiss B, Steinberger D, et al. Enhancer deletions of the SHOX gene as a frequent cause of short stature: the essential role of a $250 \mathrm{~kb}$ downstream regulatory domain. J Med Genet (2009) 46:834-9. doi:10.1136/jmg.2009.067785

17. Binder G, Renz A, Martinez A, Keselman A, Hesse V, Riedl SW, et al. SHOX haploinsufficiency and Leri-Weill dyschondrosteosis: prevalence and growth failure in relation to mutation, sex, and degree of wrist deformity. JClin Endocrinol Metab (2004) 89:4403-8. doi:10.1210/jc.2004-0591

Conflict of Interest Statement: The authors declare that the research was conducted in the absence of any commercial or financial relationships that could be construed as a potential conflict of interest.

Copyright $\odot 2018$ Lucchetti, Prontera, Mencarelli, Sallicandro, Mencarelli, Cofini, Leonardi, Stangoni, Penta and Esposito. This is an open-access article distributed under the terms of the Creative Commons Attribution License (CC BY). The use, distribution or reproduction in other forums is permitted, provided the original author(s) and the copyright owner are credited and that the original publication in this journal is cited, in accordance with accepted academic practice. No use, distribution or reproduction is permitted which does not comply with these terms. 